\title{
IMPORTANCIA DE LOS PECES EN LA NUTRICIÓN DE LA NUTRIA GIGANTE DE RÍO (PTERONURA BRASILIENSIS) (CARNIVORA: MUSTELIDAE) EN COLOMBIA
}

\author{
Ricardo Álvarez-León \\ Fundación Maguaré. Manizales (Caldas) Colombia \\ alvarez_leon@hotmail.com
}

Manizales, 2008-10-06 (Rev. 2009-11-02)

\section{RESUMEN}

Con base en la ejecución de proyectos de investigación básica y aplicada sobre de la nutria gigante de río (Pteronura brasiliensis) en Colombia, específicamente en los departamentos del Amazonas, Caquetá, Meta y Vichada, se tuvo la oportunidad única de conocer la dieta piscívora de una de las especies más representativa de la fauna acuática colombiana.

\section{PALABRAS CLAVE}

Pteronura brasiliensis, Nutria gigante de río, Trofodinámica, Peces, Etología, Orinoquia, Amazonia, Colombia.

\section{IMPORTANCE OF THE FISH DIET FOR GIANT RIVER OTTERS (PTERONURA BRASILIENSIS) (CARNIVORA: MUSTELIDAE) IN COLOMBIA}

\begin{abstract}
Based on the execution of basic and applied research projects on the giant river otter (Pteronura brasiliensis) in Colombia, specifically in the Amazonas, Caquetá, Meta y Vichada Departments, the fish diet of one of the most representative aquatic species in Colombian was studied.
\end{abstract}

\section{KEY WORDS}

Pteronura brasiliensis, giant river otter, trophodinamic, fishes, ethology, Orinoquia, Amazon, Colombia.

\section{INTRODUCCIÓN}

Con el ánimo de conocer el estado de las poblaciones de fauna silvestre de un número representativo de especies acuáticas colombianas, se inició, a partir de 1994 y en la Fundación Omacha, el proyecto Pteronura brasiliensis, el cual tuvo como objetivo general conocer, conservar y aprovechar sustentablemente este recurso de la fauna silvestre colombiana, con la distribución equitativa de los beneficios del manejo de la misma.

Respecto al conocimiento general de la especie, sobresalen los trabajos de su biología y su conservación (Carter y Rosas, 1997), estudios poblacionales, preferencias alimenticias y comportamiento en cautiverio (Carter et al., 1999), ecología y desarrollo en los ríos de Surinam (Duplaix, 1980), niveles mercurio total y metilmercurio en peces alimentos, de su dieta en Perú (Gutleb et al., 1997), ecología trófica (Rosas et al., 1999), estado y uso del hábitat y conservación en el Perú (Schenck y Stabib, 1992). 
El proyecto de la Fundación Omacha ha incluido trabajos tanto de los investigadores adscritos a la fundación como de tesistas, los cuales han permitido avanzar en el conocimiento de la especie en dos áreas fundamentales de su distribución en Colombia.

Los temas de las investigaciones institucionales han incluido algunos aspectos sobre la ecología y el hábitat de la nutria gigante en el río Caquetá medio (Amazonia) (Beltrán et al., 1994); divulgativo sobre la especie (Gómez-Serrano, 1999b). Recientemente, se justificó la inclusión del perro de agua, Pteronura brasiliensis, en la Serie de Libros Rojos de Especies Amenazadas de Colombia (Trujillo-González et al., 2006).

Así mismo, dentro de las tesis, los aspectos tratados han incluido aportes sobre el uso del hábitat y anotaciones sobre su comportamiento en el río Meta (Orinoquia) y el Caquetá medio (Amazonia) (Martínez-Arias, 1998); ecología alimentaria, en el bajo río Bita, Vichada (Orinoquía) (Gómez-Serrano, 1999a); tamaño poblacional y aspectos grupales en el bajo río Bita, Vichada (Orinoquia) (Valbuena-Vargas, 1999); abundancia, distribución y uso del hábitat en el río Orinoco, Vichada (Orinoquia) (Botello-Castillo, 2001); ecología y comportamiento en la región del bajo río Apoporis (Amazonia) (Botello-Castillo, 2000); uso del hábitat y comportamiento en el río Orinoco, Vichada (Orinoquia) (Carrasquilla, 2002); valoración biológica y cultural en el área de influencia de Puerto Carreño, Vichada, Colombia (Ríos Orinoco, Bita, Caños Juripe y Negro) (Velasco-Gómez, 2004).

Los registros y comentarios sobre su ecología en Colombia incluyen censos en el Parque Nacional Natural Tuparro (Defler, 1982), la asociación de delfines y nutrias (Defler, 1983), estado de la especie en el PNN Tuparro (Defler, 1986), comentarios sobre la legislación colombiana sobre nutrias (Donadio, 1978), estado de la especie en Colombia (Hildebrand, 1975). Más recientemente, algunos estudios preliminares sobre biología molecular, tanto en los aspectos citogenéticos (Góngora y Bernal, 1999), como citogenética, hormonal y hematológico (Góngora et al., 2000) han sido realizados y constituyen un avance considerable.

\section{RESULTADOS}

\section{Investigaciones realizadas in-situ}

Después de ejecutar ocho de los proyectos de investigación básica y aplicada, fue posible evaluar los hábitos alimenticios de la especie (Tabla 1) en cuatro Departamentos de Colombia (Amazonas, Caquetá, Meta y Vichada) y en diferentes aspectos (Biología, Ecología, Cacería, Comercialización).

Tabla 1. Peces hallados en la dieta de Pteronura brasiliensis en la Orinoquia y la Amazonia colombianas. Fuentes: (1) Martínez-Arias (1998), (2) Gómez-Serrano (1999a), (3) Gómez-Serrano (1999b), (4) Botello-Castillo (2000), (5) Botello-Castillo (2001), (6) Carrasquilla (2002), (7) Carrasquilla y Trujillo (2004), (8) Gómez-Serrano (2004), (9) Velasco-Gómez (2004).

\begin{tabular}{|l|l|l|}
\hline FAMILIAS / ESPECIES & CUENCA & FUENTES \\
\hline Anostomidae & Orinoquía & 6,7 \\
\hline Anostomus sp. & Orinoquía & $2,3,8$ \\
\hline Leporinus agassizi & Amazonia & 1 \\
\hline Leporinus fasciatus & Amazonia & $1,2,3,8$ \\
\hline Leporinus nattereri & Orinoquía & $2,3,8$ \\
\hline Leporinus spp. & Amazonia, Orinoquía & $2,3,4,5,8,9$ \\
\hline Auchenipteridae & Orinoquía & 6,7 \\
\hline Tatia sp. & Orinoquía & 9 \\
\hline Trachycoryste insignis & Orinoquía & $2,3,8$ \\
\hline Characidae & Orinoquía & 6,7 \\
\hline Acestrorynchus falcirrostris & Orinoquía & $2,3,8$ \\
\hline Acestrorynchus sp. & Orinoquía & $2,3,8$ \\
\hline Aphyocharax alburnus & Orinoquía & $2,3,8$ \\
\hline Astyanax bimaculatus & Orinoquía & $2,3,8$ \\
\hline Brycon melanopterus & Amazonia & 1 \\
\hline
\end{tabular}




\begin{tabular}{|c|c|c|}
\hline Brycon spp. & Amazonia, Orinoquía & $1,2,3,4,5,8$ \\
\hline Colossoma sp. & Orinoquía & $2,3,8$ \\
\hline Curimata sp. & Orinoquía & 9 \\
\hline Metynnis sp. & Orinoquía & $2,3,8$ \\
\hline Mylossoma sp. & Orinoquía & $2,3,8$ \\
\hline Salminus sp. & Orinoquía & $2,3,8$ \\
\hline Triportheus angulatus & Orinoquía & $6,7,9$ \\
\hline Cichlidae & Orinoquía & 6,7 \\
\hline Aequidens sp. & Orinoquía & $2,3,8,9$ \\
\hline Astronotus ocellatus & Amazonia & 4,5 \\
\hline Cichla ocellaris & Orinoquía & $2,3,8$ \\
\hline Cichla temensis & Orinoquía & $2,3,8$ \\
\hline Cichla sp. & Orinoquía & $2,3,8,9$ \\
\hline Chalceus sp. & Orinoquía & $2,3,8$ \\
\hline Crenicichla lenticulata & Orinoquía & $2,3,8$ \\
\hline Geophagus sp. & Amazonia, Orinoquía & $2,3,4,5,8$ \\
\hline Mesonauta festivum & Amazonia, Orinoquía & $1,2,3,8$ \\
\hline Mylossoma duriventris & Orinoquía & 11 \\
\hline \multicolumn{3}{|l|}{ Ctenoluccidae } \\
\hline Boulengerella sp. & Orinoquía & $2,3,6,7,8$ \\
\hline Ctenoluccius sp. & Orinoquía & $2,3,8$ \\
\hline \multicolumn{3}{|l|}{ Curimatidae } \\
\hline Curimata sp. & Orinoquía & 9 \\
\hline \multicolumn{3}{|l|}{ Cynodontidae } \\
\hline Rhaphiodon vulpinus & Orinoquía & 6,7 \\
\hline Hydrolycus scombroides & Orinoquía & $2,3,6,7,8,9$ \\
\hline Doradidae & Orinoquía & 6,7 \\
\hline Agamyxis albomaculatus & Orinoquía & 9 \\
\hline Oxydoras niger & Amazonía & $4,5,9$ \\
\hline \multicolumn{3}{|l|}{ Electrophoridae } \\
\hline Electrophorus electricus & Orinoquía & 9 \\
\hline \multicolumn{3}{|l|}{ Erythrinidae } \\
\hline Hoplias malabaricus & Amazonia, Orinoquía & $1,2,3,4,5,6,7,8,9$ \\
\hline Hoplerythrinus unitaeniatus & Amazonia, Orinoquía & $1,6,7$ \\
\hline Loricariidae & Orinoquía & 6,7 \\
\hline Ancistrus sp. & Orinoquía & $2,3,8$ \\
\hline Hoplosternum sp. & Orinoquía & $2,3,8$ \\
\hline Hypostomus sp. & Orinoquía & $2,3,8$ \\
\hline Peckoltia vittata & Orinoquía & 9 \\
\hline \multicolumn{3}{|l|}{ Osteoglossidae } \\
\hline Osteoglossum bicirrosum & Orinoquía & $2,3,8,9$ \\
\hline Osteoglossum sp. & Orinoquía & $2,3,8$ \\
\hline \multicolumn{3}{|l|}{ Pimelodidae } \\
\hline Leiarius marmoratus. & Orinoquía & $2,3,8,9$ \\
\hline Pimelodus sp. & Orinoquía & $2,3,6,7,8$ \\
\hline Pseudoplatystoma fasciatum & Amazonia, Orinoquía & $2,3,4,5,8$ \\
\hline Pseudopimelodus sp. & Orinoquía & $2,3,6,7,8$ \\
\hline \multicolumn{3}{|l|}{ Potamotrygonidae } \\
\hline Potamotrygon sp. & Orinoquía & $2,3,8$ \\
\hline \multicolumn{3}{|l|}{ Prochilodontidae } \\
\hline Prochilodus mariae & Orinoquía & 9 \\
\hline Prochilodus reticulatus & Orinoquía & $2,3,8$ \\
\hline Prochilodus sp. & Orinoquía & $2,3,6,7,8$ \\
\hline Semaprochilodus laticeps & Orinoquía & $2,3,8$ \\
\hline Semaprochilodus sp. & Orinoquía & 9 \\
\hline \multicolumn{3}{|l|}{ Serrasalmidae } \\
\hline Mylossoma duriventris & Orinoquía & $2,3,8,9$ \\
\hline Piaractus brachypomus & Orinoquía & 9 \\
\hline Pigocentrus sp. & Orinoquía & $6,7,8,9$ \\
\hline Serrasalmus spp. & Amazonia, Orinoquía & $2,3,4,5,6,7,8,9$ \\
\hline \multicolumn{3}{|l|}{ Sciaenidae } \\
\hline Plagioscion squamosissimus & Amazonia, Orinoquía & $4,5,6,7,9$ \\
\hline Plagioscion surinamensis & Orinoquía & $2,3,8$ \\
\hline \multicolumn{3}{|l|}{ Symbranchidae } \\
\hline Symbranchus sp. & Orinoquía & $2,3,8$ \\
\hline
\end{tabular}




\section{Investigaciones realizadas ex-situ}

En la actualidad sólo se tiene noticia de dos proyectos relacionados con la especie: 1) En el Zoológico de Cali (G. Corredor, com. pers.) y 2) En la Granja Piscícola Langostinos del Caribe (J. Alvarez-Barrera, com. pers.). Los ejemplares procedentes del medio natural de las cuencas del Amazonas, las que se encuentran en Cali (Valle) y del Orinoco, que se halla en Restrepo (Meta). Los trabajos han incluido diferentes aspectos de su biología, etología, ecología, comportamiento trófico y reproductivo en cautiverio. Los proyectos han sido desarrollados por investigadores y tesistas de varias universidades nacionales.

\section{DISCUSIÓN}

Los resultados de las investigaciones tróficas fueron obtenidos gracias a la observación de los hábitos alimenticios de la nutria gigante, así como de la recolección de los restos dejados por la especie, especialmente en sus letrinas y, sólo incidentalmente, en sus madrigueras.

Las 64 especies (33 válidas y 29 a nivel de género), están agrupadas en 7 órdenes y 18 familias, por lo que se considera una muy buena representación de la ictiofauna de la Orinoquía y la Amazonia colombianas.

Los órdenes muestran una amplia diversidad respecto a sus familias: Rajiformes (Potamotrygonidae), Osteoglossiformes (Osteoglossidae), Characiformes (Anostomidae, Ctenoluccidae, Characidae, Curimatidae, Cynodontidae, Prochilondontidae, Serrasalmidae), Gymnotiformes (Erythrinidae), Siluriformes (Auchenipteridae, Doradidae, Electrophoridae, Loricariidae, Pimelodidae), Symbranchiformes (Symbranchidae), y Perciformes (Cichlidae, Sciaenidae), donde sobresalen los Characiformes con ocho familias.

Aunque Gómez-Serrano (1999a, 199b, 2004), cita a Trachycoristes insignis, (Auchenipteridae) al parecer se debe a un error, pues dicha especie es de la cuenca del Magdalena-Cauca (Miles, 1947; Dahl, 1971). Revisados los trabajos de Lacambra y Pinilla (2004), Maldonado-Ocampo (2004) y Lasso-Alcalá et al. (2005), que son las más reciente recopilaciones de la Orinoquía, dicha especie no aparece, aunque hay 14 géneros registrados para la cuenca (Ageneiosus, Asterophysus, Auchenipterichthys, Centromochlus, Entomocorus, Epapterus, Gelanoglanis, Tatia, Tetranematichthys, Trachelyichthys, Trachelyopterichthys, Trachelyopterus), lo cual respalda nuestro comentario.

La cita de Prochilodus reticulatus (Prochilodontidae) (Gómez-Serrano, 1999a, 1999b, 2004), también parece ser un error, pues dicha especie es de la cuenca del Catatumbo (Galvis-Vergara et al., 1997). Revisados los trabajos de Lacambra y Pinilla (2004), Maldonado-Ocampo (2004) y Lasso-Alcalá et al. (2005) que son las más recientes recopilaciones de la Orinoquía, dicha especie no aparece para la zona.

Los peces son las presas más importantes de la dieta de las nutrias gigantes en Suramérica, las cuales consumen peces de los órdenes Characiformes, Perciformes y Siluriformes, principalmente, tal como registran: Duplaix (1980) en Surinam, Best (1984) en la región amazónica, Laidler (1984) en Guyana, Schenck (1996) en Perú, Carter y Rosas (1997) y Rosas et al. (1999) en Brasil, Carrera (2003) en Ecuador, y Martínez-Arias (1998), Gómez-Serrano (1999a, 1999b, 2004), Botello-Castillo (2000, 2001), Carrasquilla (2002), Carrasquilla y Trujillo-González (2004), Velasco-Gómez (2004) en Colombia.

La captura de los peces al parecer la inicia la hembra, la cual regula los desplazamientos de la familia (Carrasquilla, 2002; Carrasquilla y Trujillo-González, 2004); estos autores encontraron diferencias entre las capturas realizadas en el río (Siluriformes y Characiformes) y en las lagunas y tributarios (Characiformes, Perciformes y Siluriformes); así mismo, encontraron diferencias en cuanto a las familias, de las cuales Doradidae y Loricariidae sólo se encontraron en el río, en cambio Erythrinidae, Cichlidae, Auchenipteridae, Sciaenidae y Ctenoluccidae sólo se encontraron en lagunas y tributarios. 
Gómez-Serrano (1999a, 2004) comprobó la dieta preferentemente piscívora, pero encontró que también aves (Aramides cajanea, Eurypyga helias, Jacana jacana, Zenaida auriculata), reptiles (Boa constrictor, Iguana iguana) y mamíferos (Agouti paca, Dasyprocta punctata, Didelphys marsupialis, Philander opossum, Sylvilagus sp., Sciurus sp.), hacen parte de sus presas. Al relacionar las presas identificadas y los diferentes períodos hidrológicos, el citado autor concluye que las nutrias gigantes son oportunistas y consumen peces en un $96.4 \%$, principalmente de las familias Erythrinidae, Pimelodidae y Serrasalmidae. Velasco-Gómez (2004), además de la dieta preferencialmente piscívora de las nutrias gigantes, registra también en ella otros reptiles (babillas, lagartos y tortugas).

Los estudios realizados constituyen un valioso aporte al conocimiento de la nutria gigante de río (Pteronura brasiliensis) que, como se sabe, está incluida dentro de la fauna amenazada de Colombia y, por lo tanto, figura en los Libros Rojos que desde el 2002 vienen publicándose por parte de la comunidad científica y el Gobierno Nacional (TrujilloGonzález et al., 2006).

\section{CONCLUSIONES}

El análisis de la ictiofauna acompañante, que hace parte principal de la dieta de la nutria gigante, permitió conocer particularidades, semejanzas y diferencias entre las diferentes familias y especies de peces, así como su abundancia relativa y la distribución geográfica de dichas especies.

Los estudios sobre la nutria gigante y aquellos que se hallan en curso o se desarrollen en el futuro, permitirán en el corto plazo: 1) actualizar el inventario de las especies de peces incluidas en su dieta; 2) conocer el flujo genético de las diferentes poblaciones y las posibles conexiones entre subpoblaciones; 3) ampliar el conocimiento sobre algunas poblaciones y su estado sanitario; 4) definir el estado y comportamiento de las poblaciones más importantes en la Orinoquia y la Amazonia colombianas y 5) desarrollar estrategias de conservación, tanto nacionales como internacionales, y aportar bases suficientemente respaldadas para el manejo previsto por CITES.

\section{AGRADECIMIENTOS}

A las entidades y a las comunidades que apoyaron, con su conocimiento ancestral, el desarrollo de los trabajos de campo, laboratorio y gabinete, sin los cuales habría sido imposible realizar este trabajo representativo sobre la ictiofauna colombiana.

\section{BIBLIOGRAFÍA}

- $\quad$ BELTRÁN, S., J. A. DÍAZ, F. TRUJILLO \& H. DIGNUM. 1994. Algunos aspectos sobre la ecología y el hábitat de la nutria gigante de río Pteronura brasiliensis en el Caquetá medio colombiano, pp. 51 In: XIMÉNEZ, X. \& P. C. SIMOES-LÓPES (eds.) VI Reun. Espec. Mamíferos Acuáticos de América del Sur. FINEP / FBPN / BR PETROBRAS / UNEP, Florianopolis (Brasil) Outubro 24-28. Univ. Fed. Santa Catarina, $142 \mathrm{p}$.

- $\quad$ BEST, R. C. 1984. The aquatic mammals and reptiles of the Amazon. Monographic Biol., 56: 371-412.

- BOtEllo-CASTillo, J. C. 2000. Ecología y comportamiento del lobo de río Pteronura brasiliensis en la región del bajo río Apoporis, Amazonia colombiana. Tesis Profesional. Fac. de Ciencias. Univ. del Valle, 64 p.

- FBOTELlo-CASTILLO, J. C. 2001. Abundancia, distribución y uso del hábitat del perro de agua, Pteronura brasiliensis en el río Orinoco, Municipio de Puerto Carreño, Vichada. Fundación OMACHA. Bogotá D. C. Inf. Técnico.

- CARRASQUILLA, M. C. 2002. Uso de hábitat, comportamiento y dieta de la nutria gigante (Pteronura brasiliensis) en río Orinoco, Vichada, Colombia. Tesis Profesional. Fac. de Ciencias. Univ. de Los Andes, 62 p. 
- CARRAsquilla, M. C. \& F. TRUJILLO-GONZÁLEZ. 2004. Uso de hábitat, comportamiento y dieta de la nutria gigante (Pteronura brasiliensis) en río Orinoco, Vichada, Colombia, pp. 179-202 In: DÍAZGRANADOS-PITTI, M. C. \& F. TRUJILLO-GONZÁLEZ (eds.) Estudios de fauna silvestre en ecosistemas acuáticos en la Orinoquia colombiana. Fundación Omacha / IIRBAvH / GTZ / Pontificia Univ. Javeriana - IDEADE / DET. Santa Fe de Bogotá D. C. (Colombia), Serie Investigación, 6, 403 p.

- CARTER, S. K. \& F. C. W. ROSAS. 1997. Biology and conservation of the giant otter Pteronura brasiliensis. Mammal. Rev., 27 (1): 1-26.

- CARTER, S. K., F. C. W. ROSAS, A. B. COOPER \& A. C. CORDEIRO-DUARTE. 1999. Consumption rate, food preferences and transit time of captive giant otter Pteronura brasiliensis: Implications for the study of wild populations. Aquatic Mammals, 25 (2): 79-90.

- DALH, G. 1971. Los peces del norte de Colombia. MinAgricukltura - INDERENA. Bogotá D. E., 371 p.

- DEFLER, T. 1982. A census of Pteronura brasiliensis in El Tuparro National Park and environs, Colombia. INDERENA-Dir. Parques Nacionales. Bogotá D. E. Inf. Técnico, $20 \mathrm{p}$.

- DEFLER, T. 1983. Association of the giant otter Pteronura brasiliensis with fresh water dolphins (Inia goofrensis). J. Mammal., 64, 692.

- DEFLER, T. 1986. The giant otter in Tuparro National Park, Colombia. Oryx, 20: 87-88.

- DONADIO, A. 1978. Somme comments on otter trade and legislation in Colombia, pp. 34-43 In: DUPLAIS, N. (ed.) Proc. First Working Meeting of the Otter Specialist Group. Paramaribo (Suriname), March 27-29, 1977. IUCN. Publication New Series. Switzerland, $63 \mathrm{p}$.

- DUPLAIX, N. 1980. Obsevations on the ecology and behavior of the giant river otter Pteronura brasiliensis in Suriname. Rev. Ecol. (Terre et Vie), 34: 495-620.

- GALVIS-VERGARA, G., J. I. Mojica-Corso \& M. Camargo. 1997. Peces del Catatumbo. Asoc. Cravo Norte (ECOPETROL / OXY / SHELL). D’Vinni Editorial Ltda. Santa Fe de Bogotá D. C. (Colombia), 118 p.

- GÓMEZ-SERRANO, J. R. 1999a. Ecología alimentaria de la nutria gigante (Pteronura brasiliensis) en el bajo Río Bita Vichada, Colombia. Tesis Profesional. Fac. de Ciencias. Pontificia Univ. Javeriana. 50 p.

- GÓMEZ-SERRANO, J. R. 1999b. La nutria gigante de río. Gimnasio Campestre / Fundación Omacha. Santa Fe de Bogotá D. C. (Colombia), 15 p.

- GÓMEZ-SERRANO, J. R. 2004. Ecología alimentaria de la nutria gigante (Pteronura brasiliensis) en el bajo Río Bita Vichada, Colombia, pp. 203-224 In: DÍAZGRANADOS-PITTI, M. C. \& F. TRUJILLO-GONZÁLEZ (eds.) Estudios de fauna silvestre en ecosistemas acuáticos en la Orinoquia colombiana. Fundación Omacha / IIRBAvH / GTZ / Pontificia Univ. Javeriana - IDEADE / DET. Santa Fe de Bogotá D. C. (Colombia), Serie Investigación, 6, 403 p.

- GÓNGORA, J. H. \& J. E. BERNAL. 1999. Estudio citogenético de la nutria gigante de río. El Astrolabio Rev. de Invest. y Ciencia, 1 (1), 7-11.

- GÓNGORA, J. H. y otros. 2000. Estudio citogenético, hormonal y hematológico de dos nutrias gigantes de río. El Astrolbio Rev. de Invest. y Ciencia, 2 (1), 14-17.

- GutleB, A. C., C. SCHENCK \& E. STAIB. 1997. Giant river otter Pteronura brasiliensis at risk? Total mercury and methyl mercury levels in fish and otter scats, $\begin{array}{llll}\text { Peru. } & \text { Ambio, } & 26 & \text { (8): }\end{array}$ HILDEBRAND, P. VON. 1975. El perro de agua (Pteronura brasiliensis). Univ. de Los Andes. Bogotá D. E. Inf. Técnico, 12 p.

- LACAmBRA, C. \& G. PINILlA-ARANGO. 2004. Caracterización general de la ictiofauna en el área de influencia del complejo Caño Limón, Arauca, pp. 265-301 In: DÍAZGRANADOS-PITTI, M. C. \& F. TRUJILLO-GONZÁLEZ (eds.) Estudios de fauna silvestre en ecosistemas acuáticos en la Orinoquia colombiana. Fundación Omacha / IIRBAvH / GTZ / Pontificia Univ. Javeriana - IDEADE / DET. Santa Fe de Bogotá D. C. (Colombia), Serie Investigación, 6, 403 p.

- LAIDLER, L. 1984. The behavioural ecology of the giant otter in Guyana. Thesis PhD. Univ. of Cambridge, 295 p. 
- LASSO-ALCALÁ, C., J. I. y otros, 2005. Peces de la cuenca del río Orinoco. Parte I: Lista de especies y distribución por subcuencas. Biota Colombiana, 5 (2): 95158.

- MAldonAdo-OCAMPo, J. A. 2004. Peces de la Orinoquia colombiana: Una aproximación al estado actual de su conocimiento, pp. 303-368 In: DÍAZGRANADOS-PITTI, M. C. \& F. TRUJILLO-GONZÁLEZ (eds.) Estudios de fauna silvestre en ecosistemas acuáticos en la Orinoquia colombiana. Fundación Omacha / IIRBAvH / GTZ / Pontificia Univ. Javeriana - IDEADE / DET. Santa Fe de Bogotá D. C. (Colombia), Serie Investigación, 6, 403 p.

- MARTínEZ-ARIAS, A. M. 1998. Algunos aspectos del uso del hábitat de la nutria gigante de río Pteronura brasiliensis (Gmelin, 1788) y anotaciones sobre su comportamiento en el río Meta, Caquetá medio, Amazonia colombiana. Tesis Profesional. Fac. de Biol. Marina. Univ. de Bogotá Jorge Tadeo Lozano, 107 p.

- MILES, C. 1947. Los peces del río Magdalena. $1^{a}$ Edición. MinEconomía Nacional.. Bogotá (Colombia), 214 p. + Anexos.

- $\quad$ ROSAS, F. C. W., J. A. S. ZUANON \& S. K. CARTER. 1999. Feeding ecology of the giant otter Pteronura brasiliensis. Biotropica, 31 (3): 502-506.

- $\quad$ SCHENCK, C. 1996. Vorkpmmen, habitatnutzung und Schulz des Riesenotters (Pteronura brasiliensis) in Perú. Ph. D. Dissertation. Munich Universitat.

- $\quad$ SCHENCK, C. \& E. STABIB. 1992. Status, habitat use and conservation of giant otter in Peru, Chap. 20 pp. 359-370 In: Dunstone, N. \& M. L. Gorman (eds.) Behavior and Ecology of Riparian Mammals, Simp. Zool., Soc. of London 71. Cambridge. Univ. Press.

- TRUJILLO-GONZÁLEZ, F., J. C. BOTELLO-CASTILLO \& M. C. CARRASQUILLA. 2006. Perro de agua, Pteronura brasiliensis (Orden Carnivora - Familia Mustelidae), pp. 133-138 In: RODRÍGUEZ-MAHECHA, J. V., M. ALBERICO, F. TRUJILLO-GONZÁLEZ \& J. JORGENSON (eds.) Libro rojo de los mamíferos de Colombia. Serie Libros Rojos de Especies Amenazadas de Colombia. Cl-Colombia / MAVDT. Bogotá D. C. (Colombia), 433 p.

- VALBUENA-VARGAS, R. 1999. Tamaño poblacional y aspectos grupales de la nutria gigante (Pteronura brasiliensis) en el bajo río Bita, Vichada, Colombia. Tesis Profesional. Fac. de Ciencias. Pontificia Univ. Javeriana, $65 \mathrm{p}$.

- VELASCO-GÓMEZ, D. M. 2004. Valoración biológica y cultural de la nutria gigante (Pteronura brasiliensis) en el área de influencia de Puerto Carreño, Vichada, Colombia (Ríos Orinoco, Bita, Caños Juripe y Negro). Tesis Profesional. Fac. de Estudios Ambientales y Rurales. Pontificia Univ. Javeriana, 89 p. 